\title{
Fitting the ex-Gaussian equation to reaction time distributions
}

\author{
MICHAEL R. W. DAWSON \\ University of Alberta, Edmonton, Alberta, Canada
}

\begin{abstract}
Two programs that can be used to determine the probability distributions of reaction times are detailed. The first program takes rank-ordered reaction times as input and outputs a file of quantized data. The second program uses a simplex procedure to estimate the parameters of the ex-Gaussian equation that provides the best description of the quantized data. The advantages of this type of data analysis are also discussed.
\end{abstract}

Reaction time is one of the most common dependent measures used to study cognition and perception. Typically, researchers attempt to determine the effect of manipulations by analyzing differences between average response times. However, other types of analyses are possible, and in many instances are preferable (e.g., Luce, 1986; McGill, 1963; Townsend \& Ashby, 1983). For example, one may investigate effects of manipulations on the probability distributions of observed reaction times. This requires that reaction time distributions be described with mathematical equations.

The analysis of reaction time distributions is recommended on several grounds. First, response latencies are intrinsically variable, and probability distributions provide precise descriptions of this variability. Second, the shape of reaction time distributions can be used to make inferences about the identity of underlying psychological processes (McGill, 1963). Third, analyses of reaction time distributions can falsify models that are consistent with the mean and variance of response latencies (Ratcliff $\&$ Murdock, 1976).

Although the analysis of reaction time distributions is clearly desirable, a survey of the literature provides few demonstrations of how this analysis is accomplished. The purpose of this paper is to describe two computer programs that can be used to fit a particular mathematical equation to reaction time data. These programs are written in BASIC for the Commodore 64/128, but can easily be modified for use with other machines. The first program uses 8,612 bytes of RAM, whereas the second uses 10,422 bytes. The first program summarizes and condenses a large number of reaction times into a much smaller set of numbers to input to the second program. As a result, very large data sets can be analyzed. For example, Dawson and Conforto (1987) used these programs to analyze the data from 3 subjects, who had each generated 960 reaction time responses.

This research was supported by a York University Faculty of Arts Grant and by a York University President's NSERC Grant awarded to the author. Correspondence should be addressed to Michael Dawson, Centre for Advanced Study in Theoretical Psychology, University of Alberta, Edmonton, AB T6G 2E9, Canada.
The paper proceeds as follows: First, the properties of the mathematical equation to be fit to the data are described. Second, two data analysis stages are discussed in detail.

\section{THE EX-GAUSSIAN DISTRIBUTION}

The total time taken to make a response (e.g., to detect the presence of a target) comprises the time taken for many different processing stages to be completed. For instance, the target must be perceived, a decision must be made about how to respond, and then the motor activities for the response must be performed. Restle (1961) estimated that a single reaction time summarizes the latencies of 100 different component stages. The Restle estimate is highly dependent upon a specific model, and most researchers believe the number of independent components to be far fewer.

It is quite common to partition the overall reaction time into two different components or stages, the time taken to decide upon a response and the time taken to perceive the stimulus and to physically make the response (Luce, 1986, chap. 4). The former is called the decision component, and the latter is called the transduction component.

Some researchers have assumed that the reaction times from the transduction component are distributed normally (with a mean of mu and a standard deviation of sigma) and that reaction times from the decision component are distributed exponentially (with a mean of tau). This assumption is defended on the following grounds: First, the transduction component is thought to be normally distributed because it summarizes a large number of stages. Second, because most reaction time distributions are positively skewed, one of their components must be a dominant exponential distribution. Third, the exponential distribution is thought to represent the decision stage, because this component has been found to be relatively insensitive to manipulations that should affect stimulus transduction (Hohle, 1965).

The distributional assumptions described above lead to the claim that an overall reaction time for a particular trial is the sum of two reaction times, one randomly sampled 
from the transduction component distribution and the other randomly sampled from the decision component distribution. Therefore, the distribution of a set of overall reaction times can be described as the convolution of a normal distribution with an exponential distribution. This convolution results in a distribution called the ex-Gaussian distribution (Burbeck \& Luce, 1982; Luce, 1986). Mathematically, the ex-Gaussian equation is written as:

$$
\begin{aligned}
f(t) & =\frac{1}{\tau} \exp \left\{-\frac{(t-\mu)}{\tau}+\frac{\sigma^{2}}{\tau^{2}}\right\} \\
& \times \Phi\left\{\frac{t-\mu}{\sigma}-\frac{\sigma}{\tau}\right\}
\end{aligned}
$$

where $\Phi$ is normal CDF.

The ex-Gaussian distribution is an attractive choice for describing response latencies. First, it has been found to give very good descriptions of reaction time data (Dawson \& Conforto, 1987; Hockley, 1982, 1984; Hohle, 1965; Ratcliff, 1978, 1979; Ratcliff \& Murdock, 1976). Second, the three parameters that define an ex-Gaussian distribution (mu, sigma, and tau) provide an explicit account of the distributional properties of the two different processing components.

In fitting an ex-Gaussian distribution to data, two different steps must be considered. First, the data must be summarized. Second, the ex-Gaussian distribution that best describes the summarized data must be obtained by estimating values for the three parameters. These steps are considered below.

\section{PROGRAM 1: VINCENT AVERAGING}

The first step in the analysis is to summarize the reaction time data such that its distributional properties become evident. Ratcliff (1979) described one method of doing this, called Vincent averaging. Vincent averaging serves two purposes. First, it quantizes data in such a way that the profile of the data distribution can be plotted. Second, it permits the creation of an average data set that preserves information contained in the profiles of its constituents.

The algorithm for Vincent averaging a single data set is as follows: (1) Rank order the set of $N$ reaction times from lowest to highest. (2) Choose the desired number of quantiles $(N Q)$ to describe the data. (3) Create a list of numbers that is $N \times N Q$ entries long by writing each reaction time in the original list $N Q$ times. (4) Add the first $N$ entries in this list, and divide by $N$. This is the value of the first quantile. (5) Repeat Step 4 for the next $N$ numbers in the list to calculate the value of the next quantile; repeat until the end of the list of numbers is reached. A Vincentized data set describing a group of files can be created by averaging the quantiles of several data sets that have been treated in this manner.

VINCENT is a program that Vincent averages sets of reaction times. It is assumed that each set of reaction times has already been rank ordered and saved as an individual data file. The user specifies how many of these data files are to be input, indicates the maximum length of these files, and types in the names of the files to be read. A variable name is also assigned to each of these data sets. Each of the specified files is input individually, and the algorithm described above is applied to calculate the quantiles for each data set. After each file has been processed, the average quantiles are calculated and are associated with the variable name "TOTAL." The quantized data sets (individual and average) are then saved in a single sequential file. This file is read by the second program, described below.

Vincentized data can be plotted as a set of histograms (Ratcliff, 1979). The edges of the histograms are drawn at each quantile. The height of each histogram is determined by realizing that the areas of all of the histograms are equal, because the data has been split into equal quantiles. In the program described below, the area of each histogram has been set to .05 units, because the data is being treated as a probability distribution with a total area of 1.00. Therefore, the height of the histogram drawn from quantile $N$ to quantile $N+1$ is equal to .05/(Quantile $N+1$ - Quantile $N$ ). Ratcliff presents a number of examples of this type of representation.

\section{PROGRAM 2: FITTING THE EX-GAUSSIAN}

Once the reaction times have been Vincentized, an exGaussian distribution can be fit to the data. This requires estimating the three parameters that define the ex-Gaussian distribution, such that some measure of fit between the equation and the Vincentized data is minimized. EXGAUSSIAN SIMPLEX is a program that estimates the parameters by minimizing the chi-square fit between the data and the model.

The first main step in the program is to read a file of Vincentized data. A file created by the Vincent averaging program is input, and the variable names associated with each quantized data set are displayed. The user selects which of the quantized data sets are to be fit by the exGaussian distribution. Thus one can examine the averaged data or any of the individual data sets in the file.

A simplex optimization method (Adby \& Dempster, 1974) is used to find the best values of tau, mu, and sigma. In general, this technique moves a geometric shape (called a simplex) through a space defined by the parameters to be estimated. If there are $N$ parameters to be estimated, then the simplex will have $N+1$ vertices, whose coordinates are particular values of the parameters being estimated. The algorithm for moving the simplex from an initial starting point in the parameter space to the place in the parameter space that produces the function minimum is as follows: (1) Evaluate the function at each simplex point. (2) Find the point that produces the maximum function value. (3) Determine the centroid of all of the other simplex points. (4) Reflect, by some step size, the point that produced the maximum function value through the centroid. This creates a new position for the simplex. 
These steps are then repeated. At various points in this iterative process, the step size is decreased. Eventually, all of the points in the simplex will converge on the function minimum.

The particular algorithm used in Program 2 is the simplex method described by Nelder and Mead (1965). In addition to following the general steps described above, this algorithm allows the volume of the simplex to be expanded or contracted to make the search through the parameter space more efficient. The first step in this procedure is to input the initial estimates of the three parameters in order to provide a starting point for the simplex. These estimates should be in units of seconds; the sum of mu and tau should be approximately equal to the data mean, and sigma should be relatively small. For example, in the analyses reported by Dawson and Conforto (1987), the starting point was tau $=.100, \mathrm{mu}=.300$, and sigma $=.010$ for data with means in the order of 400 msec.

The next step in the procedure is to specify the parameters for adjusting the simplex and the criterion for convergence. The default values are recommended by Nelder and Mead (1965). Next, the initial simplex is created. Finally, the points of the simplex are iteratively evaluated and shifted through the parameter space. The function that is evaluated is written as a subroutine in the program, and is described in more detail below. For specific information about the operations performed during each iteration, the reader is referred to the algorithm flowchart provided by Nelder and Mead (1965, p. 309).

At the end of each iteration, a convergence index is calculated by a call to subroutine 600 . This index is the standard error of the function values calculated for each simplex point. If the index is below a present criterion (default value equal to $1.0 \mathrm{E}-10$ ), then the iterations stop and the final estimates of the parameters are sent to the printer. In addition, the program prints the values for each quantile, the observed height of the histograms at each quantile, and the estimated height of the histograms at each quantile (derived from the ex-Gaussian equation). This printout can also be generated at any time during iterative processing by pressing the $\mathrm{F} 1$ function key.

During each iteration, the function that is minimized is evaluated for at least one simplex point. (In order to speed up processing, points that have not changed position from one iteration to the next are not reevaluated.) This is done by passing the parameter values that define a simplex point to the subroutine that defines the function being evaluated. At each quantile, this subroutine calculates the chi-square fit between the observed height of the data histograms and the height predicted from the exGaussian equation using the simplex point coordinates as parameters.

This latter calculation is done in two stages. The leftmost part of Equation 1 is evaluated directly. The cumulative normal part of Equation 1 is estimated by using the Hastings approximation recommended by Bock and Jones $(1968$, p. 339). The two components of the ex-Gaussian equation are then combined and used in the calculation of the chi-square value that measures the fit with respect to all of the quantiles. As three parameters are being estimated, the expected chi-square is equal to the number of quantiles minus 3 (e.g., Glass \& Hopkins, 1984). If this value is exceeded, then the ex-Gaussian equation differs significantly from the data being described.

One drawback to the program is that it is extremely slow to converge. One analysis typically takes several hours to complete. To alleviate this problem somewhat, the analysis can be performed in two stages. In the first stage, set the convergence criterion fairly high so that the program converges quickly to values that are rough approximations of the best parameter estimates. These values can then be used as the starting point for a more detailed analysis with a more stringent convergence criterion. If this approach is taken, the initial simplex in the second stage should be made smaller by changing the 0.1 in Line 234 to 0.01 .

\section{CONCLUDING REMARKS}

These two programs provide a convenient means for using a small computer to describe the probability distributions of reaction times. They have been successfully used to analyze the data obtained in studies of visual search (Dawson \& Conforto, 1987). Of course, the programs can be modified to suit the needs of individual users. One particularly interesting type of modification is possible in Program 2. By changing the function described in subroutine 500 (and by adjusting the number of parameters processed), other types of distributions can be fit to reaction time data. (For descriptions of other promising distributions, see Luce, 1986.) As more computer programs of this type become available, distributional analyses of reaction time data will become more and more common.

\section{AVAILABILITY}

Readers who are interested in obtaining copies of these programs are invited to send a blank diskette to the author. Readers who are interested in using these programs on machines other than the Commodore $64 / 128$ can write to the author to obtain listings of the programs in BASIC.

\section{REFERENCES}

ADBY, P. R., \& DEMPSTER, M. A. H. (1974). Introduction to optimization methods. New York: Wiley.

Bock, R. D., \& Jones, L. V. (1968). The measurement and prediction of judgment and choice. San Francisco: Holden-Day.

Burbeck, S. L., LuCE, R. D. (1982). Evidence from auditory simple reaction times for both change and level detectors. Perception \& Psychophysics, 32, 117-133.

Dawson, M. R.W., \& Conforto, D. (1987). The independent processing of features and of locations during visual perception. Manuscript submitted for publication.

GLASS, G. V., \& HoPKINS, K. D. (1984). Statistical methods in education and psychology. Englewood Cliffs, NJ: Prentice-Hall.

HOCKLEY, W. E. (1982). Retrieval processes in continuous recogni- 
tion. Journal of Experimental Psychology: Leaming, Memory, \& Cognition, 8, 497-512.

HoCKLEY, W. E. (1984). Analysis of response time distributions in the study of cognitive processes. Journal of Experimental Psychology: Learning, Memory, \& Cognition, 10, 598-615.

HoHLE, R. H. (1965). Inferred components of reaction times as functions of foreperiod duration. Journal of Experimental Psychology, 69, 382-386.

LuCE, R. D. (1986), Response times. New York: Oxford University Press.

McGill, W. J. (1963). Stochastic latency mechanisms. In R. D. Luce, R. R. Bush, \& E. Galanter (Eds.) Handbook of mathematical psychology (Vol. 1, pp. 309-359). New York: Wiley.

Nelder, J. A., \& MEAD, R. (1965). A simplex method for function minimization. The Computer Journal, 7, 308-313.
Ratcliff, R. (1978). A theory of memory retrieval. Psychological Review, 85, 59-108.

RATCLIFF, R. (1979). Group reaction time distributions and an analysis of distribution statistics. Psychological Bulletin, 86, 446-461.

RatClifF, R., \& MURDOCK, B. B. (1976). Retrieval processes in recognition memory. Psychological Review, 83, 190-214.

Restle, F. (1961). Psychology of judgment and choice: A theoretical essay. New York: Wiley.

TownsEnd, J. T., \& AsHBY, F. G. (1983). Stochastic modeling of elementary psychological processes. New York: Cambridge University Press.

(Manuscript received July 24, 1987; revision accepted for publication September 22, 1987.) 